\title{
Satisfação e Qualidade de Vida em Profissionais de Enfermagem na Região do Cariri Cearense
}

\author{
Josefa Janiele Lopes Moreira ${ }^{1}$; Juliana de Freitas Vasconcelos Sugette ${ }^{2}$; Martha Maria Macedo Bezerra ${ }^{3}$
}

\begin{abstract}
Resumo: A Satisfação com a vida e, a Qualidade de vida no trabalho, nos parece estar cada vez mais prejudicada em função de mudanças por que passa o mundo nos tempos atuais. Diversos fatores e uma série de responsabilidades recaem sobre o labor do profissional de educação, muitos dos quais fogem ao seu controle. $\mathrm{O}$ presente estudo se propôs a conhecer e analisar, em que medida a Satisfação com a Vida e a Qualidade de Vida dos professores da Escola Municipal Padre José de Anchieta, variam em função de variáveis com a remuneração, carga horária semanal de trabalho, formação acadêmica dos mesmos, dentre outras. Trata-se de um estudo transversal, quali-quantitativo, correlacional, do tipo ex post facto. Considerou-se a correlação de um conjunto de variáveis antecedentes (Nível de formação acadêmica, Remuneração, Jornada de trabalho) com as variáveis critério (Satisfação com a Vida e Qualidade de Vida). Os resultados se mostraram preocupantes, quando $16 \%$ dos professores se disseram insatisfeitos em exercerem o seu trabalho como educadores. Quanto ao motivo das insatisfações, os baixos salários foi citado por $88 \%$ dos entrevistados, seguidos de estrutura escolar deficiente, desmotivação, dificuldade de educar, atrito de ideais, desvalorização da categoria e, a falta de incentivo. A preocupação com a qualidade de vida docente, torna-se relevante, na medida em que professores quando satisfeitos, produzem mais, influenciam na motivação dos seus alunos e, principalmente, ajudam a construir uma sociedade melhor e mais justa.
\end{abstract}

Palavras-chave: Qualidade de vida, Satisfação com a vida, Professor.

\section{Satisfaction and Quality of Life in Nursing Professionals in the Cariri Region of Ceará}

\begin{abstract}
Satisfaction with life and quality of working life seems to be more and more damaged given the changes in the world nowadays. Several factors and series of responsibilities rely on educational professional's labor, where some of them may escape from professional control. The present work propose to know and analyze to what degree satisfaction with life and quality of life of teachers in Escola Municipal Padre José de Anchieta vary according to variables such as salary, weekly hours worked, academic background, etc. It's a cross research, qualitativequantitative study, ex post facto. It was considered the correlation of previous variables in a set (academic background level, salary, work load) with criteria variables (Satisfaction with life and quality of life). The results shown concerning, when $16 \%$ of teachers said they're unsatisfied in doing their jobs as educators. About the reason of unsatisfying, low salaries was quoted by $88 \%$ of interviewees, followed by deficiency on educational structure, demotivation, difficulty on educate, ideals grip, devaluation of the own working-class and the lack of encouragement. The concerning with quality of teacher's life become relevant in that teachers, when satisfied, may produce more, influence student's motivation and above all help to build a better and more fair society.
\end{abstract}

Keywords: quality of life, satisfaction with life, teacher.

\footnotetext{
${ }^{1}$ Graduação em Medicina pela Universidade Federal do Ceará (2013). Atualmente é médica do Programa Saúde da Família na Prefeitura Municipal de Crato-CE. Contato: anie-moreira@ hotmail.com;

${ }^{2}$ Graduação em Medicina pela Universidade Federal do Ceará (2015), atualmente residente do segundo ano de Clínica Médica do Hospital Universitário Walter Cantídio. No momento, trabalha na uti adulto do Hospital Universitário Walter Cantídio, uti adulto do Hospital Antônio Prudente, médico do setor de emergência do Hospital Geral de Fortaleza e time de resposta rápida (TRR) do Hospital do Coração de Messejana. Contato: julianafv@hotmail.com;

${ }^{3}$ Mestrado em Educação pela Universidade Estadual do Ceará (2013) Possui Licenciatura em Pedagogia pela Universidade Regional do Cariri (1994), especialização em Língua Portuguesa pela Universidade Estadual do Ceará (1995), especialização em Políticas Públicas pela Universidade Regional do Cariri (2001), especialisação em Saude Mental e Psiquiatria pela Universidade Estadual do Vale do Acaraú (2013). Atualmente, cursa o Doutorado em Saúde Coletiva pela Faculdade de Medicina do ABC. Professora concursada da Secretaria de Educação do Estado do Ceará. Professora da Rede Municipal de Juazeiro do Norte. Contato: marthamacedo2016@gmail.com.
} 


\section{Introdução}

A Satisfação no trabalho e, mais especificamente a Satisfação laboral em enfermeiros, são estudos escassos e, relativamente bem recentes. Alguns deles tendem a aparecer associados à personalidade, ou à motivação, ou ainda a algum mal-estar profissional.

Estudos da década de 70 e década de 80, parecem iniciar um direcionamento sobre o assunto, porém relacionando a qualidade de vida a satisfação laboral, envolvendo aspectos do meio familiar e da saúde mental (SECO, 2000).

O conceito de satisfação laboral, normalmente é relacionado `a Psicologia Social ou Organizacional. Na Psicologia Social, a satisfação laboral é definida como uma interrelação de sentimentos negativos ou positivos, manifestado pela pessoa em relação ao seu trabalho (SECO, 2000). Carlotto (2011) associa a satisfação laboral como uma associação dos componentes afetivos a autoestima e ao trabalho que se realiza numa organização.

Gursel, Sunbul e Sari (2002) definem a satisfação laboral, como um estado emocional resultante do trabalho profissional, associado às tarefas próprias da profissão. Este construto seria exteriorizado pela dedicação e forma como o indivíduo de se comporta no ambiente de trabalho.

Qualidade de vida (QV), de acordo com a Organização Mundial da Saúde, é compreendida como uma percepção indivídual de posição na vida, em determinado contexto cultural e de valores, onde se vive. Tal expressão se refere também ao momento de vida de cada um na sociedade, inclua-se aí, os momentos de trabalho, e sua percepção da qualidade de vida no trabalho (QVT), já que não seria possível uma dissociação de vida e trabalho (CUNHA, 2008).

Atualmente, a QVT compreende as dimensões física, psicológica, tecnológica e social, correspondendo a valores numa perspectiva de organização mais saudável e humana (CUNHA, 2008). Neste caso, a satisfação dos indivíduos em um ambiente laboral seguro, envolvido em relações de respeito mútuo, e com relativas oportunidades de desenvolvimento profissional (SCHMIDT, DANTAS e MARZIALE, 2008). Considera-se, para efeito deste estudo, uma caracterização satisfatória da QVT, valorização do trabalhador, a sua participação em colegiados de decisão na empresa, o incentivo ao seu desenvolvimento e manifestação de seu 
potencial criativo, boas condições laborais e de desenvolvimento de relações humanas de trabalho (FELLI e TRONCHIN, 2005).

Não parece haver um consenso quanto a conceito ou ainda sobre qual seria a melhor maneira de se avaliar a QVT ou satisfação laboral, no caso dos trabalhadores da Enfermagem (FELLI e TRONCHIN, 2005). Muitos estudos disponíveis nesta área específica, tem abordado a QV de pacientes ou de seus familiares, havendo pouca frequência em estudos mais específicos sobre a QVT de enfermeiros. Outra constatação é que, dentre os estudos realizados, há predominância de ênfase nas questões patológicas, incluindo-se aí, principalmente o adoecimento e questões ligadas a fatores de risco para esta categoria profissional de saúde, especialmente no ambiente hospitalar (CAMPOS e DAVID, 2007).

A avaliação de ambientes laborais revela possíveis questões ligadas a (in)satisfação com a profissão exercida. A ocorrência de muitas doenças emocionais, tem sido associada a questões do trabalho e, é assunto estudado em vários países. A síndrome de Burnout, também tem sido frequentemente, associada à insatisfação laboral e, causadora de baixo rendimento, absenteísmo, problemas de qualidade na produtividade, e redutora da Satisfação com a Vida em profissionais da saúde (CARLOTTO, 2004; 2011).

Estudos realizados confirmam um consenso de que a enfermagem é uma ocupação altamente desgastante, com grandes repercussões na saúde emocional e física, além de afetar o desempenho desses trabalhadores (CARLOTTO, 2011).

Na Região Nordeste do Brasil, tem sido possível perceber estudos sobre situações de estresse, bem como de insatisfação no trabalho em trabalhadores da saúde, notadamente os da Enfermagem (OLIVEIRA et.al., 2009; OLIVEIRA et.al., 2013; BARROSO et.al., 2015), que indicam a pouca atenção às suas próprias condições de cuidado com a saúde. É fato que os ambientes de postos de saúde ou hospitalares, são tidos como ambientes com frequente tensões, tanto para os trabalhadores em saúde quanto para os usuários (OLIVEIRA et.al., 2009). Neste contexto, observam-se novas e diversas questões de saúde, nem sempre de fácil lida ou de rápida resolução. Isso implica em desafios diversos envolvendo as inúmeras necessidades e demandas advindas do processo de atendimento.

Diante desta situação, nos indagamos: qual o nível de Satisfação laboral e Qualidade de Vida em profissionais de enfermagem na Região do Cariri Cearense? 
Neste sentido o objetivo principal consistiu em conhecer e analisar, em que medida a Satisfação com a Vida e a Qualidade de Vida dos profissionais de enfermagem de um hospital público de referência no município de Juazeiro do Norte, estado do Ceará, variam em função das variáveis: remuneração, carga horária semanal de trabalho, número de plantões em média e, formação acadêmica dos mesmos.

Para isso foi necessário também, analisar o nível de (in)satisfação dos profissionais de enfermagem de um hospital público de referência no município de Juazeiro do Norte, com vida e com a realização do seu trabalho; conhecer sobre a satisfação com a vida desses enfermeiros; conhecer os motivos dos Enfermeiros insatisfeitos, em continuarem na profissão e, discutir sobre a Qualidade de vida dos sujeitos quanto aos domínios físico, psicológico, social e ambiental.

\section{Método}

Estudo transversal, quali-quantitativo e correlacional. Considerou-se a correlação das variáveis antecedentes, a exemplo de Nível de formação acadêmica, Remuneração e Jornada de trabalho, com variáveis critério, a exemplo de Satisfação com a vida e, Qualidade de Vida.

As questões de natureza qualitativa disseram respeito à percepção dos sujeitos quanto à permanência na profissão de enfermeiro, motivos que os impulsionam a continuarem na profissão e causas da insatisfação laboral, se este for o caso.

Estas questões foram analisadas segundo a técnica da análise de conteúdo. Segundo Vala (1986), uma das técnicas mais comuns em pesquisas empíricas realizadas em diferentes ciências humanas e sociais. Esse tipo de análise permite inferências sobre a organização do sistema de pensamento dos sujeitos.

Bardin (2001), afirma ser a análise de conteúdo:

\footnotetext{
"um conjunto de técnicas de análise das comunicações visando obter, por procedimentos sistemáticos e objetivos de descrição do conteúdo das mensagens, indicadores (quantitativos ou não) que permitem a inferência de conhecimentos relativos às condições de produção/recepção (variáveis inferidas) destas mensagens..." (p 42).
} 
Vala (1986) comenta os aspectos conceituais da análise de conteúdo abordada por diferentes autores, afirmando que:

“... o caráter objetivo e sistemático da análise de conteúdo referido por Berelson e Cartwright e as condições de validade e replicabilidade expressa por Krippendorff enquanto técnica de pesquisa, a análise de conteúdo exige a maior explicitação de todos os procedimentos utilizados ..." (p.103).

Assim sendo, esta técnica, enquanto analisa as condições de produção da comunicação pelo sujeito, efetua inferências sobre as mensagens inventariadas e sistematizadas, articulando as falas com o contexto de produção.

O estudo foi realizado em um hospital de referência estadual na região do Cariri cearense, mais especificamente no município do Juazeiro do Norte.

A amostra compreendeu, vinte e cinco profissionais entre enfermeiros e técnicos de enfermagem, que se dispuseram a responder ao questionário proposto. $\mathrm{O}$ instrumento constou das seguintes partes: Uma Escala de Satisfação com a Vida (DIENER et al.,1985), com o propósito de avaliar o julgamento das pessoas acerca da satisfação com suas vidas. Trata-se de uma escala que apresenta um só fator, justamente a Satisfação com a Vida. É simples e de rápida aplicação, além de ser utilizada em diversos países (PAVOT, DIENER, 1993); a Escala de Qualidade de Vida WHOQOL-bref da Organização Mundial de Saúde (WHO, 1998), cujas medidas fornecem informações pessoais, sociais e de bem-estar psicológico (HIGGINSON et al, 2001). Apesar das dificuldades, tais avaliações podem nos proporcionar um melhor entendimento sobre as necessidades das pessoas em seus ambientes laborais (FLECK et al, 1999); Bem como Informações sócio-demográficas, com o objetivo de caracterizar a amostra, a exemplo de sexo, idade, escolaridade, Renda, número de plantões, se possui insatisfações no seu trabalho como profissional de saúde, quais os motivos que os faz permanecerem na profissão. 
Id on Line Revista Multidisciplinar e de Psicoloqia

Id on Line Multidisciplinary and Psycology Journal

\section{Resultados e Discussões}

\section{Perfil geral dos sujeitos}

Fizeram parte deste estudo 25 profissionais da enfermagem de um hospital de referência estadual em Juazeiro do Norte, estado do Ceará, sendo 10 enfermeiros e 15 técnicos de enfermagem.

A seguir, a distribuição dos sujeitos de acordo com o sexo e a idade.

Tabela 1: Distribuição do sexo e idade dos participantes da pesquisa

\begin{tabular}{|c|c|c|c|c|}
\hline Sexo & & \multicolumn{2}{|c|}{ Freqüência } & Percental (\%) \\
\hline Masculino & & \multicolumn{2}{|c|}{5} & 20 \\
\hline Feminino & & \multicolumn{2}{|c|}{18} & 72 \\
\hline Não responderam & & \multicolumn{2}{|c|}{2} & 8 \\
\hline Total & & \multicolumn{2}{|c|}{25} & 100,0 \\
\hline & Mínimo & Máximo & Média & Desvio padrão \\
\hline Idade & 20 & 54 & 30,2 & 8,421 \\
\hline
\end{tabular}

Juazeiro do Norte-CE, 2016

As idades dos sujeitos variaram entre 20 e 54 anos (média 30,2; Dp=8,421), a maioria do sexo feminino, dezoito participantes $(72,0 \%)$, e cinco do sexo masculino (20,0\%). Dois respondentes evitaram a questão $(8 \%)$.

Quanto à renda familiar, os participantes auto referiram o seguinte: quinze participantes (60\%) recebem entre 2 a 3 salários mínimo vigente; seis (24\%), de 4 a 5 salários mínimo vigente e, quatro participantes (16\%) referiram mais do que cinco salários mínimo vigente. O salário mínimo à época do estudo era de $\mathrm{R} \$ 880,00$.

Todo o trabalho é gerador de fatores desgastantes e potencializadores de estresse, que são determinantes dos processos saúde-doença vivenciados pelos trabalhadores e da satisfação e qualidade de vida no trabalho. (ROCHA e FELLI, 2004).

Foi indagado sobre o quão sentem-se satisfeitos com o seu trabalho. Os sujeitos responderam de acordo com o demonstrado na tabela 3, a seguir. 
Tabela 3: Satisfação com o trabalho como Profissional de Saúde na Enfermagem

\begin{tabular}{lcc}
\hline & Freqüiência & Percentual (\%) \\
\hline SIM & 21 & 84 \\
\hline NÃO & 4 & 16 \\
\hline Total & $\mathbf{2 5}$ & $\mathbf{1 0 0 , 0}$ \\
\hline
\end{tabular}

Juazeiro do Norte-CE, 2016

Neste caso, 21 respondentes (84\%) relataram sentirem satisfação com seu trabalho na enfermagem e, quatro (16\%), ao contrário, não se sentem satisfeitos em exercerem o seu trabalho como profissionais da enfermagem.

Quanto aos motivos das insatisfações, os baixos salários foi citado por 22 dos participantes (88\%), seguidos de estrutura material deficiente, desmotivação, dificuldade com os colegas de trabalho, desvalorização da categoria e, a falta de incentivo.

Indagados sobre o por quê de ainda permanecerem na profissão, os resultados foram os seguintes: 17 sujeitos (68\%) referiram que amam o que fazem; 5 referiram que gostam de trabalhar na profissão (20\%); um referiu a falta de outros mercados de trabalho (4\%); um sujeito (4\%) referiu que precisa pagar as contas e sustentar a família e, outro (4\%) relatou possuir identidade com a profissão, por ser formado na área.

A satisfação que sentimos no trabalho é uma variável importante para os estudos organizacionais. Autores como Staw e Ross (1985) ressaltam tratar-se de uma das variáveis mais comumente estudadas na área da Psicologia Organizacional. Martinez (2002) explicou em seus estudos que, que no século $\mathrm{XX}$, o construto satisfação era comumente relacionado ao processo motivacional, e as teorias de Maslow, de 1970, e de McGregor de 1960.

Siqueira (1995), por sua vez, advoga que os estudiosos da temática começaram a defender a satisfação como uma causa de comportamentos no trabalho refletindo no desempenho, rotatividade, produtividade, bem como no absenteísmo.

Ainda não há um consenso acerca da definição e da satisfação no trabalho. Weiss (2002) conceituou o satisfação no trabalho como um julgamento positivo ou negativo que fazemos sobre nosso trabalho ou determinada situação no trabalho. Valle (2007), por sua vez, afirma, que a maioria dos determinantes da satisfação no trabalho implica em um sistemas de 
recompensas, que incluem desde a distribuição e centralização de poder, até a autoestima e a necessidade de realização.

Siqueira e Gomide Júnior (2004) expõem que, a satisfação parece ser considerada como uma reação a vários componentes do trabalho, que podem desencadear no indivíduo sentimentos de satisfação ou insatisfação. Assim seriam fontes de satisfação no trabalho, o próprio trabalho, o local adequado, os salários e as oportunidades de promoção.

Em resumo, os sujeitos, neste estudo parecem ter associado à satisfação no trabalho com diversas dimensões percebidas como inadequadas, como infra-estrutura, salários e outros benefícios, mesmo que parte dessa situação se deva ao próprio profissional, como o salário por exemplo, que é maior, quanto maior for a sua titulação e habilidade percebida. E as próprias reações dos clientes, muitas vezes se dá em função da carga de desmotivação do profissional, que é percebida, refletindo-se esta em ações pouco estimulantes.

Percebe-se também uma certa ambiguidade nas respostas, uma vez que, mesmo relatando insatisfação com os baixos salários $(88 \%)$ dos profissionais, esses mesmos referem que continuam na profissão por amor ao que fazem ou porque gostam da profissão (88\%).

Os sujeitos foram solicitados a responderem se existem políticas públicas, aplicadas na saúde, em seu local de trabalho, que sejam motivadoras para os profissionais de enfermagem, as respostas foram as seguintes:

Tabela 4: Existem políticas públicas, aplicadas no seu local de trabalho, que sejam motivadoras para os profissionais de saúde?

\begin{tabular}{lcc}
\hline Existem políticas públicas motivadoras? & Freqüiência & Percentual (\%) \\
\hline $\begin{array}{l}\text { SIM - Há no hospital algum incentivo, apesar } \\
\text { das dificuldades }\end{array}$ & 19 & 76 \\
\hline SIM - Há cursos de formação propostos & 2 & 8 \\
\hline NÃO - Tudo são exigências & 1 & 4 \\
\hline NÃO & 3 & 12 \\
\hline Total & $\mathbf{2 5}$ & $\mathbf{1 0 0 , 0}$ \\
\hline
\end{tabular}

Juazeiro do Norte-CE, 2016 
A Tabela 4 mostra que 21 professores (84\%) acreditam que há políticas públicas aplicadas no hospital, que são, de alguma maneira percebidas como motivadoras para os profissionais da enfermagem. Ao passo que 4 respondentes não o perceberam dessa forma $(16 \%)$

Sobre esse sentido, Porto e Tamayo (2003), bem como Paz (2004), já haviam ressaltado que o trabalho tende a contribuir para auto-estima, convívio social e saúde mental dos trabalhadores. Tamayo (2004) observou que o tempo dedicado ao trabalho é um componente fundamental para a satisfação laboral e para a própria motivação.

Políticas públicas que privilegiem o trabalho, atendendo aos desejos humanos de sentir conforto para produzir no seu trabalho, certamente serão altamente geradoras de satisfação no trabalho. Dessa forma o indivíduo tenderá a se ver como um membro produtivo da sociedade, cumprindo seu papel social.

\section{Satisfação com a vida dos participantes}

No tocante a Satisfação com a vida, nas dimensões da escala, as respostas estão indicadas na tabela a seguir:

Tabela 5: Satisfação com a vida dos participantes da amostra.

\begin{tabular}{l|c|c|c|c|c|c|c}
\hline & $\begin{array}{c}\text { Discordo } \\
\text { Totalmente } \\
(1)\end{array}$ & $\begin{array}{c}\text { Discordo } \\
(2)\end{array}$ & $\begin{array}{c}\text { Discordo } \\
\text { Ligeiramente } \\
(3)\end{array}$ & $\begin{array}{c}\text { Nem } \\
\text { Concordo } \\
\text { nem } \\
\text { Discordo } \\
(4)\end{array}$ & $\begin{array}{c}\text { Concordo } \\
\text { Ligeiramente } \\
(5)\end{array}$ & $\begin{array}{c}\text { Concordo } \\
(6)\end{array}$ & $\begin{array}{c}\text { Concordo } \\
\text { Totalmente } \\
(7)\end{array}$ \\
\hline $\begin{array}{l}\text { 1.Na maioria dos } \\
\text { aspectos, minha } \\
\text { vida é próxima ao } \\
\text { meu ideal. }\end{array}$ & - & $8,0 \%$ & $8,0 \%$ & $16,0 \%$ & $36,0 \%$ & $28,0 \%$ & $4,0 \%$ \\
\hline $\begin{array}{l}\text { 2.As condiços da } \\
\text { minha vida são } \\
\text { excelentes }\end{array}$ & $8,0 \%$ & $8,0 \%$ & $16,0 \%$ & $12,0 \%$ & $24,0 \%$ & $28,0 \%$ & $4,0 \%$ \\
\hline $\begin{array}{l}\text { 3.Estou satisfeito } \\
\text { (a) com minha } \\
\text { vida }\end{array}$ & - & $4,0 \%$ & $20,0 \%$ & $20,0 \%$ & $24,0 \%$ & $24,0 \%$ & $8,0 \%$ \\
\hline $\begin{array}{l}\text { 4.Dentro do } \\
\text { possível, tenho } \\
\text { conseguido as } \\
\text { coisas } \\
\text { importantes que } \\
\text { quero na vida }\end{array}$ & - & $8,0 \%$ & $8,0 \%$ & $12,0 \%$ & $36,0 \%$ & $28,0 \%$ & $8,0 \%$ \\
\hline
\end{tabular}


Id on Line Revista Multidisciplinar e de Psicoloqia

Id on Line Multidisciplinary and Psycology Journal

\begin{tabular}{|c|c|c|c|c|c|c|c|}
\hline $\begin{array}{l}5 . \text { Se pudesse } \\
\text { viver uma } \\
\text { segunda vez, não } \\
\text { mudaria quase } \\
\text { nada na minha } \\
\text { vida }\end{array}$ & $12,0 \%$ & $16,0 \%$ & $28,0 \%$ & $8,0 \%$ & $20,0 \%$ & $12,0 \%$ & $4,0 \%$ \\
\hline
\end{tabular}

Juazeiro do Norte-CE, 2016

Apesar das condições de trabalho, muitas vezes adversas que os profissionais de saúde parecem enfrentar, no geral as pessoas que compuseram a presente amostra relataram estarem relativamente satisfeitos com suas vidas.

Os resultados demonstraram que 68,0\% dos entrevistados acreditam que na maioria dos aspectos, sua vida é próxima do ideal e que, $56,0 \%$ dos sujeitos concordam que estão satisfeitos com a sua vida da maneira como ela é e, $56,0 \%$ dos entrevistados afirmaram que as condições de suas vidas são excelentes.

Sua pontuação média $(M=22,3$, com Desvio Padrão=5,065) está dentro da amplitude que permite classificá-los como ligeiramente satisfeitos (21 a 25 pontos), de acordo com estudos de Pavot e Diener, 1993.

Quanto às respostas dadas a cada um dos cinco itens desta medida, merece particular atenção as maiores médias, em termos de concordar totalmente, verificadas para os itens 3 - “ Estou satisfeito(a) com minha vida" e 4 - "Dentro do possível tenho conseguido coisas importantes que quero da vida". Certamente, o conteúdo destes itens refletem sentimentos de auto-realização. Acreditamos que não se trataria apenas de bens materiais conseguidos, pois, se observa no item 2 - "As condições da minha vida são excelentes", este foi o que recebeu menor pontuação e, o item 5 - "Se pudesse viver uma segunda vez, não mudaria quase nada na minha vida" foi pontuado como discordante por $56 \%$ dos respondentes.

A satisfação com a vida foi afetada pelo sexo dos respondentes. Homens $(M=27,53$, com Desvio Padrão $=6,06)$ se mostraram mais satisfeitos com suas vidas que as mulheres $(\mathrm{M}$ $=21,32 ; \mathrm{DP}=10,34)$, para $\mathrm{t}=2,76 \mathrm{e} \mathrm{p}<0,05$. Este resultado encontra-se de acordo com $\mathrm{a}$ literatura, especializada (PAVOT, DIENER, 1993; SHEVLIN, BRUNSDEN, MILES, 1998; ARRINDELL, HEESINK, FEIJ, 1999) A pontuação de satisfação com a vida não variou em função da idade dos participantes. Concretamente, algumas pesquisas consideram que pessoas mais velhas apresentam maior satisfação com a vida (EHRLICH E ISAACOWITZ , 2002). É possível que o tamanho da amostra, (menos que 200 sujeitos) tenha influenciado os resultados. 
Em resumo, Maples (1992) nos orienta que, não podemos deixar de observar as consequências nefastas que, índices baixos de satisfação com a vida, quando encontrados em meio a profissionais de saúde, tendem a acarretar. Os sentimentos advindos da baixa satisfação com a vida, afetam os próprios profissionais da enfermagem, primeiramente e, tende a comprometer muito seriamente o clima vivenciado na equipe de trabalho e na organização como um todo. Em tese, profissionais interagem com os colegas de trabalho e com os pacientes sob seus cuidados, contribuindo diretamente para a falta de motivação e desinteresse dos mesmos. A consequência é que ambos podem experienciar uma menor qualidade de vida e, o consequente comprometimento do tratamento.

\section{Qualidade de vida dos Profissionais de Saúde.}

Com relação a auto-avaliação da Qualidade de vida, medida através da questão 1 do WOHQOL, esta foi avaliada da seguinte maneira: 4\% (1) avaliou como ruim; 24\%(6 profissionais) avaliaram como Nem ruim, nem boa; e, 72\% (18 profissionais) avaliaram sua qualidade de vida como boa.

Indagados o quão estão satisfeitos com a sua saúde (questão 2 do WOHQOL), de uma maneira geral, as respostas foram as seguintes: $4 \%$ (1 respondente) se autodeclarou insatisfeito; $16 \%$ (4 respondentes) se autodeclararam nem satisfeitos, nem insatisfeitos e, $80 \%$ (20 respondentes) se declararam satisfeitos com a sua saúde, no momento em que responderam ao questionário.

A saúde e o suporte social tem se mostrado em diversos estudos, como bons preditores de Satisfação com a vida (Viswervaran, Sanchex e Fisher, 1999). No caso do presente estudo, observou-se que, os respondentes, se perceberam como pessoas mais saudáveis (média de 103,84 no domínio físico do WOHQOL e 81,44 no domínio psicológico - Tabela 6) e satisfeitas com o trabalho que realizam como profissionais da enfermagem (84\% - Tabela 3).

Com relação aos domínios do WHOQOL abreviado, as respostas estão consolidadas na tabela a seguir: 
Tabela 6: Dimensões da Qualidade de vida dos participantes da pesquisa.

\begin{tabular}{|l|c|c|c|c|c|}
\hline & $\begin{array}{c}\text { Domínio } \\
\text { Físico }\end{array}$ & $\begin{array}{c}\text { Domínio } \\
\text { Psicológico }\end{array}$ & $\begin{array}{c}\text { Domínio } \\
\text { Social e de } \\
\text { Relações }\end{array}$ & $\begin{array}{c}\text { Domínio } \\
\text { Ambiental }\end{array}$ & $\begin{array}{c}\text { Qualidade } \\
\text { de Vida } \\
\text { Global }\end{array}$ \\
\hline Média & 103,84 & 81,44 & 42,72 & 95,84 & 80,96 \\
\hline Desvio Padrão & 14,943 & 13,55 & 4,861 & 17,832 & 10,979 \\
\hline Mínimo & 76 & 56 & 36 & 72 & 64 \\
\hline Máximo & 136 & 104 & 52 & 136 & 106 \\
\hline
\end{tabular}

Juazeiro do Norte-CE, 2016

Com relação a Qualidade de vida, medida de acordo com os parâmetros da Organização Mundial de Saúde, observou-se que em todos os domínios, os respondentes da amostra apresentaram médias superiores às médias descritas e padronizadas como normais.

Em termos do domínio físico a média segundo a OMS seria 74 pontos. Os profissionais de saúde pontuaram 103,84; no domínio psicológico, a média segundo a OMS é descrita na literatura como 70 pontos. Os profissionais pontuaram 81,44 neste domínio. Quanto ao meio ambiente, a pontuação média da OMS é de 57, na nossa amostra foi de 95,85. Quanto a qualidade de vida de uma maneira Global, a média da OMS é de 64 pontos. Na nossa amostra essa pontuação foi de 80,96 .

A evidência, obtida sugere que, o aumento da satisfação com o trabalho, parece conduzir a um aumento do bem-estar. Um estudo longitudinal de Winefield, Tiggemann e Goldney (1998) com jovens, demonstrou que um aumento da satisfação com a vida, estaria relacionada com a saúde psicológica ao longo do tempo.

Por outro lado, qualquer intervenção que promovessem um aumento na satisfação com a vida, também aumentaram o bem-estar ao longo do tempo (BARRIOS-CHOPLIN, MCCRATY e CRYER, 1997) e ainda, as emoções positivas parecem proteger os indivíduos do estresse laboral (FOLKMAN e MOSKOWITZ, 2000).

Talvez por isso, nosso estudo demonstrou que a maioria dos profissionais da amostra pareceram integrados do ponto de vista da satisfação laboral, superando as adversidades naturais da profissão de educador.

Alinhado com esse pensamento, Pereira ett all (1998) afirmam que a prática docente estaria permeada por tensões decorrentes das condições de trabalho dos profissionais de 
enfermagem, das expectativas que a sociedade tem para com o trabalho deles e, da própria imagem que possuem de si. Os fatores descritos parecem ser geradores de problemas reais como: desinteresse por estresse, falta de tempo para refletirem sobre sua prática e, queda na qualidade do seu trabalho.

Concluímos pois que, quanto ao significado da prática de enfermagem para os profissionais da amostra neste estudo, estaria mais associada a sua própria habilidade pessoal, ou seja, a uma maneira mais conveniente, que encontram para atuar em no hospital, e para lidarem com sua prática e pacientes sua responsabilidade. A prática então, não está relacionada à atividade de enfermagem em si, mas também a outras condições, percebidas pelo profissional, como remuneração adequada e políticas públicas para a saúde, etc.

No que se refere à satisfação no trabalho verificou-se que a maioria dos respondentes pesquisados estão satisfeitos com a atividade que desempenham. Para eles o seu trabalho parece gerar resultados que implicam no bem estar dos clientes, além da possibilidade de vir a desenvolver suas habilidades interpessoais e ainda, promover benefícios para a comunidade.

Essa satisfação parece fundamental para a vida dos professores, já que se passa grande parte do dia, no ambiente laboral e, não havendo satisfação, outras áreas poderão ser afetadas.

\section{Considerações Finais}

Em um mundo onde a mudança é a tônica e única realidade, onde, cada vez mais se valoriza o capital de maneira intensa, de forma a tentar assegurar a sobrevivência, a saúde se torna extremamente necessária.

A preocupação com a qualidade de vida do profissional de saúde, se apresenta como de extrema relevância, pois é nas mãos desses profissionais que nos entregamos quando em situação de crise de saúde. Para isso precisamos de seu apoio, comprometimento e amor.

Profissionais satisfeitos produzem mais, influenciam na melhora do estado geral dos pacientes e ajudam a construir um clima laboral melhor.

Feitas essas ponderações, acredita-se que os objetivos do presente estudo tenham sido alcançados. As evidências empíricas aqui reunidas e apresentadas, explicam diferenças e 
similaridades entre os correlatos demográficos e a satisfação com a vida dos profissionais que trabalham no âmbito da saúde.

Tendo em vista que o tema em pauta é muito vasto, recomenda-se outras possibilidades de estudos futuros na área, tais como:

-A realização de pesquisas sobre Qualidade de vida e/ou Satisfação com a vida dos Enfermeiros, considerando-se um número maior de instituições de saúde, buscando-se um maior conhecimento da realidade no município estudado;

- Conhecer a realidade em relação aos construtos estudados em outras cidades da Paraíba e do Nordeste do Brasil, para se identificar e comparar as diferenças regionais.

É importante sugerir também que haja maiores investimentos nos processos de trabalho dos profissionais da enfermagem, cuidando-se da infra-estrutura em termos de espaços adequados, iluminação e ventilação apropriadas, além de laboratórios ergonomicamente arranjados com controle de riscos previstos apropriadamente. Estas também são condições importantes a se levar em conta, quando tratamos da questão da qualidade de vida. Afinal, não somente o trabalho, mas a Satisfação e a Qualidade de vida são fatores que contribuem significativamente para dar sentido às nossas vidas.

\section{Referências}

ARRINDELL WA, HEESINK J, FEIJ JA. The Satisfaction With Life Scale (SWLS): Appraisal with 1700 health young adults in The Netherlands. Personality and Individual Differences, 26: 815-26, 1999.

BARDIN, Laurence. Análise de conteúdo. Lisboa: Edições 70, 2002

BARRIOS-CHOPLIN, B.; MCCRATY, R.; CRYER, B. An inner quality approach to reducing stress and improving physical and emotional well-being at work. Stress Medicine, v. 13, n. 3, p. 193-201, 1997.

BARROSO, M.L.; OLIVEIRA, G.F.; CARVALHO, A.C.F.; BATISTA, H.M.T.; SILVEIRA, G.B.M. Estresse e Uso de Álcool em Enfermeiros que trabalham em Urgência e Emergência. Caderno de Cultura e Ciência, Ano IX, v.13, n.2, Mar, 2015.

CAMPOS JF, DAVID HMSL. Abordagens e mensuração da qualidade de vida no trabalho de enfermagem: produção científica. Rev Enferm. UERJ. 2007;15(4):584-9. 
CARLOTO, C. M. Ruptura ou esforço da dominação: gênero em perspectiva. In: GODINHO, T.; SILVEIRA, M. L. (Orgs.). Políticas públicas e igualdade de gênero. São Paulo, 2004. p. 149-156. (Caderno da Coordenadoria Especial da Mulher, n. 8.

CARLOTTO, Mary Sandra. Fatores de risco da síndrome de burnout em técnicos de enfermagem. Rev. SBPH, Rio de Janeiro, v. 14, n. 2, p. 07-26, dez. 2011.

CUNHA KC, coordenador. Gestão de pessoas: foco na enfermagem atual. São Paulo: Martinari; 2008.

DIENER, E., EMMONS, R. A., LARSEN, R. J., e GRIFFIN, S. The Satisfaction With Life Scale. Journal of Personality Assessment, 1985. 49, 71-75.

EHRLICH BS, ISAACOWITZ DM. Does subjective well-being increase with age? Perspectives in Psychology, 5: 20-6, 2002.

FELLI VE, TRONCHIN DM. A qualidade de vida no trabalho e a saúde do trabalhador de enfermagem. In: Kurcgant P, coordenadora. Gerenciamento em enfermagem. Rio de Janeiro: Guanabara Koogan; 2005. p. 89-107.

FLECK, MPA; LEAL OF; LOUZADA, S; XAVIER, M; CHACHAMOVICH E, VIEIRA G et al. Desenvolvimento da versão em português do instrumento de avaliação de qualidade de vida da OMS (WHOQOL-100). Rev Bras Psiquiatr. 21(1):19-28. 1999.

FOLKMAN, S. e MOSKOWITZ, J. T. Positive affect and the other side of coping. American Psychologist, 2000. 55, 647-654.

GURSEL, M., SUNBUL, A. M., e SARI, H. An analysis of burnout and job satisfaction between Turkish headteachers and teachers. European Journal of Psychology of Education, 2002. 17, 35-45.

HIGGINSON IJ, CARR AJ. Measuring quality of life: Using quality of life measures in the clinical setting. BMJ. 2001.

MAPLES, M. F. Teachers need self-esteem too: A counseling workshop for elementary school teachers. Elementary School Guidance and Counseling, 1992. 27, 33-39.

MASLOW, A. H. Motivation and Personality, 2nd. Ed., New York, Harper \& Row. ISBN $0060419873,1970$.

McGREGOR D. The Human Side of Enterprise New York McGraw-Hill, 1960.

MRUCK, C. Auto-estima: Investigación, teoría y práctica . Bilbao: Desclée de Brouwer, 1998. 
OLIVEIRA, G. F., BARBOSA, G. A., SOUZA, L. E. C., COSTA, C. L., ARAÚJO, R. C. R., \& GOUVEIA, V. V. Satisfação com a vida entre profissionais de saúde: correlatos demográficos e laborais. Revista Bioética, 17(2), 319-334, 2009.

OLIVEIRA, N.S.; LESSA, J.F.; BARROSO, M.L.; OLIVEIRA, G.F.; BIANCO, B.A.V. O Enfermeiro e a Saúde Mental no Contexto da Estratégia Saúde da Família. Cadernos de Cultura e Ciência 12 (2), 66-77 2013.

PAVOT, W. e DIENER, E. Review of the satisfaction with life scale. Psychological Assessment, 1993. 5 (2), 164-172.

PEREIRA, A.M.T.B, Hartmann, J.B. \& Campos, L.F. A síndrome de burnout: causas e tratamento. 1998. Reunião Especial da Sociedade Brasileira para o Progresso da Ciência - SBPC, Programa \& Anais. Maringá, 6, p.12.

ROCHA, SSL e FELLI, VEA. Qualidade de vida no trabalho docente em enfermagem. Revista Latino-Americana de Enfermagem v. 12, n. 1, p. 28,35, 2004.

SCHMIDT DR, DANTAS RA, MARZIALE MH. Quality of life at work: Brazilian nursing literature review. Acta Paul Enferm. 2008; 21(2):330-7.

SECO, G. B. A satisfação na actividade docente. Coimbra: Universidade de Coimbra, 2000.

SHEVLIN, M., BRUNSDEN, V., e MILES, J. N. V. Satisfaction With Life Scale: Analysis of factorial invariance, mean structures, and reliability. Personality and Individual Differences, 1998. 25, 911-916.

SIQUEIRA, M. M. M. e GOMIDE Jr., S. Vínculos do indivíduo com o trabalho e com a organização. Em J. C. Zanelli, J. E. Borges-Andrade \& A. V. B. Bastos (Orgs), Psicologia, organizações e trabalho no Brasil (pp. 300-328). Porto Alegre: Artmed, 2004.

TAMAYO, Alvaro. Cultura e saúde nas organizações. In: FERREIRA, Maria Cristina; ASSMAR, Eveline Leal. Cultura, Satisfação e Saúde nas Organizações. Porto Alegre: Artmed, 2004.

VALLE, Vanice Regina Lírio do. Sobre um conteúdo jurisdicionalmente sindicável de políticas públicas: primeiros esforços de reflexão. Intervenção no II Congresso IberoAmericano de Direito Administrativo (evento paralelo), havida em Curitiba, maio/2007.

VISWERVARAN, C., SANCHEX, J. I. e FISHER, J. The role of social support in the process of work stress: a meta-analysis. Journal of Vocational Behaviour, 1999. 54, 314-334.

WEISS, H. M. Deconstructing job satisfaction: Separating evaluations, beliefs and affective experiences. Human Resource Management Review,2002. 12, 173-194. 
Id on Line Revista Multidisciplinar e de Psicoloqia

Id on Line Multidisciplinary and Psycology Journal

WHO (World Health Organization) 1998. WHOQOL and spirituality, religiousness and personal beliefs (SRPB). Report on WHO consultation. MNH/MAS/ MHP/98.2 WHO, Genebra. 22 pp.

Como citar este artigo (Formato ABNT):

MOREIRA, Josefa J.L.; SUGETTE, Juliana F V.; BEZERRA, Martha M.M. Satisfação e Qualidade de Vida em Profissionais de Enfermagem na Região do Cariri Cearense. Id on Line Revista Multidisciplinar e de Psicologia, Maio de 2017, vol.11, n.35, p.590-606. ISSN: 1981-1179.

Recebido: 29.05 .2017

Aceito: 31.05 .2017 